\title{
BRIEF REPORTS \\ Decreased Clearance of von Willebrand Factor in a Patient With Type 2B von Willebrand Disease Following Development of Immune Thrombocytopenia
}

\author{
Zhihong J. Wang, MD, PhD, ${ }^{1 *}$ Nkechi Onwuzurike, $\mathrm{MD}^{2}$ Michael U. Callaghan, $\mathrm{MD}^{1}{ }^{1}$ Madhvi Rajpurkar, $\mathrm{MD},{ }^{1}$ \\ Meera Chitlur, $\mathrm{MD}^{1}{ }^{1}$ and Jeanne $\mathrm{M}$. Lusher, $\mathrm{MD}^{1}$
}

We report a case of concurrent type $2 \mathrm{~B}$ von Willebrand disease (VWD) and immune thrombocytopenia (ITP). The patient had characteristic loss of von Willebrand factor (VWF) high molecular weight multimers (HMWM) but a normal platelet count in the initial 8 years after diagnosis of type 2B VWD. When he developed severe thrombocytopenia, however, both his VWD indices and VWF
HMWM normalized. As his platelet count increased, he again lost the HMWM and his VWD indices decreased. These results suggest that the severe thrombocytopenia led to decreased clearance of VWF, especially the HMWM. Pediatr Blood Cancer 2008;51:416418. (c) 2008 Wiley-Liss, Inc.

Key words: hemostasis; ITP; thrombocytopenia; VWD; VWF

\section{INTRODUCTION}

Type 2B VWD is caused by gain-of-function mutations in exon 28 of the VWF gene encoding the binding site for platelet GPIb, leading to increased affinity of VWF to platelet GPIb with subsequent increased clearance of HMWM in plasma [1-3]. The multimeric structure of VWF allows it to function as a molecular bridge between the subendothelial matrix and the platelet GPIb receptor complex [3]. One of the characteristic findings in type 2B VWD is the relative loss of VWF HMWM in plasma, which is believed to be due to increased clearance of VWF-platelet complex. Here we describe a patient with type $2 \mathrm{~B}$ VWD with characteristic loss of VWF HMWM and a normal platelet count initially. Interestingly, his HMWM appeared when he developed ITP, and disappeared again when his platelet count increased. Possible causes leading to the restoration of his HMWM are discussed.

\section{CASE REPORT}

The patient is a 13-year-old African American male who was diagnosed with type 2B VWD at 5 years of age. He presented with a history of frequent epistaxis since 6 months of age. Laboratory evaluation at diagnosis was characteristic of type 2B VWD, including decreased VWF activity (24\%), VWF antigen (0.32 U), and FVIII activity (45\%); increased low dose ristocetin-induced platelet aggregation (RIPA) and absence of VWF HMWM (Fig. 1A). He required periodic antihemophilic factor/von Willebrand factor complex (Humate-P, manufactured by ZLB Behring Gmbh, Marburg, Germany) for the management of severe epistaxis. His platelet count remained normal (242,000-292,000 $\left.\mu \mathrm{l}^{-1}\right)$ until 8 years after the diagnosis of type $2 \mathrm{~B}$ VWD, when he was found to be thrombocytopenic during the evaluation for a traumatic lip laceration. His platelet count nadir was $6,000 \mu 1^{-1}$ (Fig. 2). Further evaluation revealed an increased number of megakaryocytes in his bone marrow and autoantibodies to GPIIb/IIIa, Ib/IX and Ia/IIa in his platelet eluates. (The test for platelet auto antibodies was performed at the Blood Center of Wisconsin as previously described [4].) As ITP was suspected, he received anti-D immunoglobulin (WinRho, manufactured by Cangene Corporation, Winnipeg, Canada), prednisone and intravenous immunoglobulin therapy over the following 2 months with poor response. His platelet counts ranged from 6,000 to $24,000 \mu 1^{-1}$. Surprisingly, a repeat VWD work up 10 weeks after he developed thrombocytopenia was within normal limits. VWF activity was $66 \%$, VWF antigen was $77 \%$, FVIII activity was $93 \%$, and VWF multimeric analysis revealed the presence of a full range of VWF multimers (Fig. 1B). Of note, he had not received any VWF-containing products in the previous 2 months. To reconfirm the diagnosis of type $2 \mathrm{~B}$ VWD, a GPIb binding assay was performed as described [5], and revealed increased platelet GPIb binding to VWF, consistent with his diagnosis of type $2 \mathrm{~B}$ VWD. Additionally, he had a point mutation $(4022 \mathrm{G}>\mathrm{A})$ in exon 28 of VWF gene known to be associated with type 2B VWD [6]. His ADAMTS-13 activity was normal and there were no ADAMTS-13 inhibitors. His platelet count slowly increased to $44,000 \mu \mathrm{l}^{-1}$ 5 months following the diagnosis of ITP (Fig. 2). At that time, his VWD indices, including VWF antigen (52\%), activity (59\%), and FVIII activity (57\%) again decreased; VWF multimeric distribution was abnormal with the loss of HMWM but the presence of intermediate size multimers (Fig. 1C), which was different from his VWF multimer pattern at diagnosis. His platelet count increased to $66,000 \mu \mathrm{l}^{-1}$ approximately 2 years after he was diagnosed with ITP.

\section{DISCUSSION}

We report a patient with concurrent ITP and type 2B VWD. The normalization of his VWD indices and restoration of his VWF HMWM following the onset of thrombocytopenia are of particular interest. Type 2B VWD is characterized by the relative loss of VWF HMWM in plasma, which is believed to be due to spontaneous binding of mutated VWF HMWM to platelets, which causes platelet aggregation followed by removal of the VWF-platelet aggregates. This leads to the loss of HMWM and thrombocytopenia [1,7]. The

\footnotetext{
${ }^{1}$ Division of Hematology/Oncology, Children's Hospital of Michigan, Wayne State University, Detroit, Michigan; ${ }^{2}$ Department of Pediatrics, Hurley Medical Center, Flint, Michigan

*Correspondence to: Zhihong J. Wang, Division of Hematology/ Oncology, Children's Hospital of Michigan, 3901 Beaubien, Detroit, MI 48201. E-mail: jwang2@med.wayne.edu
}

Received 4 October 2007; Accepted 18 March 2008 
A

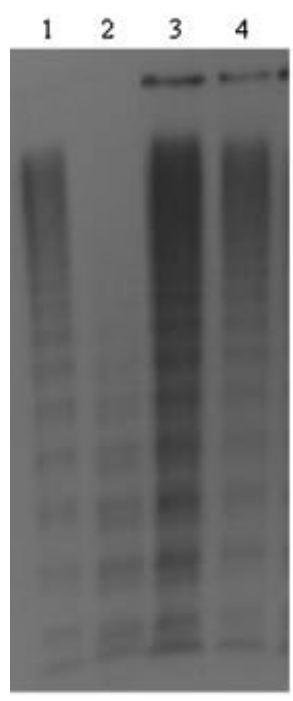

B

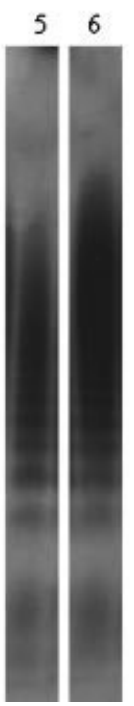

C

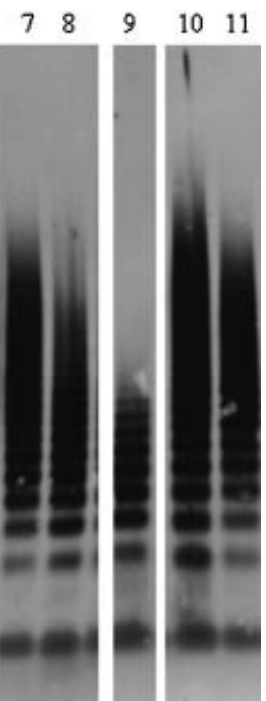

Fig. 1. VWF multimer analysis at diagnosis of type $2 B$ VWD and after the development of ITP. A: VWF multimer analysis at diagnosis. Absence of HMW VWF multimers was evident in the patient's sample. 1 and 4: Normal controls; 2: patient; 3: unknown. Multimer analysis was performed at the Mayo Clinic Coagulation Laboratory. B: VWF multimer analysis at two and half months following the development of ITP. Normal VWF multimeric distribution was seen with a full range of VWF multimers. 5: Patient; 6: normal control. Multimer analysis was performed at the Blood Center of Wisconsin. C: VWF multimer analysis 5 months after the development of ITP when platelet count increased. VWF multimeric distribution was abnormal with absence of HMWM but presence of intermediate size multimers. This pattern was not typical of type 2B vWD and differed from the pattern at diagnosis. The patient's platelet count at that time was $44,000 \mu 1^{-1} .7$ and 11: Normal controls; 8 : patient; 9: type 2B VWD control; 10: large multimer control. Multimer analysis was performed at the Blood Center of Wisconsin.

patient had a characteristic loss of VWF HMWM when his platelet count was normal, however, his HMWM were restored when he developed severe thrombocytopenia, and disappeared again when his platelet count increased. This suggests that the reduced number of platelets led to decreased clearance of VWF and restoration of VWF HMWM and provides strong evidence that the loss of VWF HMWM and thrombocytopenia in type 2B VWD is due to the increased clearance of platelet-VWF complexes (as this patient's VWF multimer pattern was restored to normal when there were essentially no platelets present).

Thrombocytopenia is a frequent finding in type 2B VWD and is believed to be due to increased clearance of VWF-platelet complexes [8]. However, the thrombocytopenia in our patient is different from the thrombocytopenia in type 2B VWD. First, he was not thrombocytopenic in the initial 8 years of follow-up. Second, when his thrombocytopenia appeared, it was severe and associated with restoration of previously absent VWD HMWM. Additionally, the presence of several autoantibodies to platelets supports the diagnosis of ITP. While his poor initial response to treatment is not typical of ITP, it is not uncommon in this age group. Furthermore, since increased clearance of VWF-platelet complex leads to thrombocytopenia in type $2 \mathrm{~B}$ VWD, it is also possible that his underlying type $2 \mathrm{~B}$ VWD may have masked his initial response to treatment.

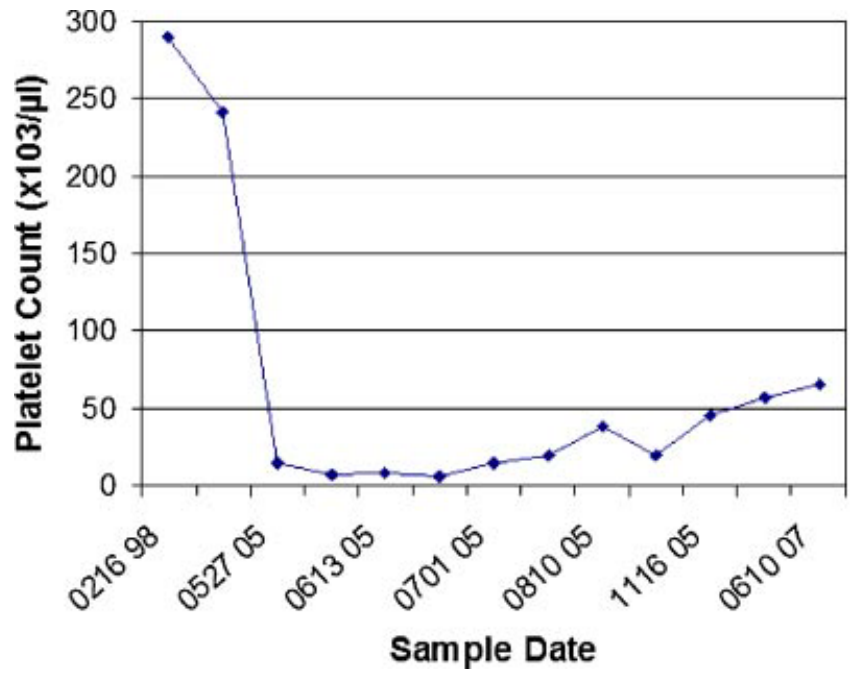

Fig. 2. Platelet count prior to and following the development of ITP. Platelet count was normal at diagnosis and remained normal $(242,000-$ $292,000 \mu 1^{-1}$ ) until the patient developed ITP 8 years after he was diagnosed with type 2B VWD. The platelet count decreased on 27 May 2005 with a nadir of $6,000 \mu \mathrm{l}^{-1}$. It started increasing 3 months after he was found thrombocytopenic and reached $66,000 \mu \mathrm{l}^{-1}$ in June 2007, approximately 2 years later.

Although speculative, another possibility is that the autoantibody to platelet GPIb present in his plasma has interfered with the binding of VWF to platelets and led to the decreased consumption of VWF. The interaction between VWF and GPIb is mediated by specific regions of both components [9]. Cooney et al. proposed that the VWF GPIb binding domain can adopt either a discrete "on" or "off" conformation [10]. Naïve VWF in the circulation is predominantly in the "off" conformation and does not associate with platelets. In type 2B VWF mutants, VWF is locked in the "on" conformation, which heightens VWF-platelet binding and spontaneous platelet aggregation, leading to increased clearance of VWF HMWM [10]. We speculate that in the patient we describe, plateletbound GPIb antibody may have caused the change of VWF conformation from the locked "on" to "off" status and modified the gain-of-function defect, and therefore, led to reduced affinity of VWF for platelets and decreased the clearance of VWF HMWM. Some published reports support this possibility $[11,12]$. The immune inhibition of RIPA by rabbit antisera to a human platelet antigen was described by Nachman et al. [11], and the inhibitory effect of antibodies on mutated VWF-induced platelet aggregation was further confirmed by the study of De Romeuf et al. [12]. Using recombinant VWF (rVWF) reproducing four type 2B VWD missense mutations, they have shown that both the binding of mutated rVWFs to platelets and the platelet aggregation induced by rVWFs are specifically inhibited by monoclonal antibodies to GPIb or VWF [12].

In summary, we have demonstrated the restoration of VWF HMWM in a patient with type 2B VWD following the onset of ITP. Our observations suggest that the reduced numbers of platelets secondary to ITP caused decreased clearance of VWF-platelet complexes. This case report provides in vivo evidence that the loss of VWF HMWM and thrombocytopenia in type 2B VWD are due to increased clearance of platelet-VWF complexes. 


\section{ACKNOWLEDGMENT}

We thank Dr. Joan Gill at the Blood Center of Wisconsin for facilitating the molecular testing; Dr. Kenneth Friedman at the Blood Center of Wisconsin and Dr. William Nichols at the Mayo Clinic Special Coagulation Laboratory for providing us with the pictures of the patient's VWF multimers. Zhihong J. Wang was a recipient of an Amgen Oncology Fellowship. Michael Callaghan is the recipient of an NHF/Baxter Clinical Fellowship.

\section{REFERENCES}

1. Federici AB, Mannucci PM. Advances in the genetics and treatment of von Willebrand disease. Curr Opin Pediatr 2002; 14:23-33.

2. Ruggeri ZM. von IWillebrand factor. Curr Opin Hematol 2003; 10:142-149.

3. Saba HI, Fujimura Y, Saba SR, et al. Spontaneous platelet aggregation in type IIB Tampa von Willebrand disease is inhibited by the $52 / 48-\mathrm{kDa}$ fragment of normal von Willebrand factor, which contains the GPIb binding domain. Am J Hematol 1989;30:150153.

4. Davoren A, Bussel J, Curtis BR, et al. Prospective evaluation of a new platelet glycoprotein (GP)-specific assay (PakAuto) in the diagnosis of autoimmune thrombocytopenia (AITP). Am J Hematol 2005;78:193-197.

5. Scott JP, Montgomery RR. The rapid differentiation of type IIb von Willebrand's disease from platelet-type (pseudo-) von
Willebrand's disease by the "neutral" monoclonal antibody binding assay. Am J Clin Path 1991;96:723-728.

6. Sadler JE, Ginsburg D. A database of polymorphisms in the von Willebrand factor gene and pseudogene. For the Consortium on von Willebrand Factor Mutations and Polymorphisms and the Subcommittee on von Willebrand Factor of the Scientific and Standardization Committee of the International Society on Thrombosis and Haemostasis. Thromb Haemost 1993;69:185-191.

7. Lankhof H, Damas C, Schiphorst ME, et al. Functional studies on platelet adhesion with recombinant von Willebrand factor type 2B mutants R543Q and R543W under conditions of flow. Blood 1997;89:2766-2772.

8. Mathew P, Greist A, Maahs JA, et al. Type 2B vWD: The varied clinical manifestations in two kindreds. Haemophilia 2003;9:137144.

9. Vicente V, Houghten RA, Ruggeri ZM. Identification of a site in the alpha chain of platelet glycoprotein $\mathrm{Ib}$ that participates in von Willebrand factor binding. J Biol Chem 1990;265:274-280.

10. Cooney KA, Ginsburg D. Comparative analysis of type $2 b$ von Willebrand disease mutations: Implications for the mechanism of von Willebrand factor binding to platelets. Blood 1996;87:23222328.

11. Nachman RL, Jaffe EA, Weksler BB. Immunoinhibition of ristocetin-induced platelet aggregation. J Clin Invest 1977;59: $143-148$.

12. de Romeuf C, Hilbert L, Mazurier C. Platelet activation and aggregation induced by recombinant von Willebrand factors reproducing four type $2 \mathrm{~B}$ von Willebrand disease missense mutations. Thromb Haemost 1998;79:211-216.

\title{
Reversible Skeletal Changes After Treatment With Bevacizumab in a Child With Cutaneovisceral Angiomatosis With Thrombocytopenia Syndrome
}

\author{
Angela R. Smith, $\mathrm{MD}_{,}{ }^{1 *}$ Jane M. Hennessy, $\mathrm{RN}, \mathrm{CNP}^{2}{ }^{2}$ Margaret A. Heisel Kurth, $\mathrm{MD}^{2}{ }^{2}$ and Stephen C. Nelson, $\mathrm{MD}^{2}$
}

Cutaneovisceral angiomatosis with thrombocytopenia (CAT) syndrome is a rare vascular disorder of the skin and gastrointestinal tract for which there is no standard treatment. We present a case in which a child with CAT syndrome was treated with bevacizumab, a vascular endothelial growth factor inhibitor, and subsequently developed asymptomatic metaphyseal bone lesions. Though not previously described as a side effect, we hypothesize that the use of bevacizumab in a child with active epiphyseal growth plates caused these radiographic lesions. Because of the potential for altered bone growth and metabolism, children receiving VEGF inhibitors should be monitored closely for bony toxicity. Pediatr Blood Cancer 2008;51:418-420. (๑) 2008 Wiley-Liss, Inc.

Key words: avastin; bevacizumab; bony toxicity; CAT syndrome

\section{INTRODUCTION}

Cutaneovisceral angiomatosis with thrombocytopenia (CAT) syndrome is a rare vascular disorder that was first recognized as a distinct entity in 2001 with a description of 10 patients with multiple skin and gastrointestinal vascular lesions associated with minor, but sustained thrombocytopenia. Histopathology of the vascular lesions in CAT syndrome shows dilated thin-walled channels with endothelial hyperplasia. The lesions have varying degrees of dilation and endothelial hyperplasia [1]. North et al. also describe three patients with similar clinical symptoms and histopathology, but have termed the entity "multifocal lymphangioendotheliomatosis with thrombocytopenia" because of its histologic similarity to benign lymphangioendothelioma which is most commonly seen as an acquired lesion in adulthood [2].
The clinical features of CAT syndrome include multiple, discrete red-brown or blue skin lesions, gastrointestinal lesions and thrombocytopenia. Unlike infantile hemangioma, the skin lesions

\footnotetext{
${ }^{1}$ Department of Pediatric Hematology/Oncology/Blood and Marrow Transplantation, University of Minnesota, MMC 484, 420 Delaware Street SE, Minneapolis, Minnesota 55455; ${ }^{2}$ Department of Pediatric Hematology/Oncology, Children's Hospitals and Clinics of Minnesota, 2525 Chicago Ave, Minneapolis, Minnesota 55404

*Correspondence to: Angela R. Smith, Fellow, Pediatric Hematology/ Oncology/Blood and Marrow Transplantation, University of Minnesota, MMC 484, 420 Delaware Street SE, Minneapolis, MN 55455. E-mail: smith719@umn.edu
}

Received 18 January 2008; Accepted 19 March 2008 